\title{
How elements up to 118 were reached and how to go beyond
}

\author{
Christoph E. Düllmann $1,2,3,4, \star$ \\ ${ }^{1}$ Institute of Nuclear Chemistry, Johannes Gutenberg University, 55128 Mainz, Germany \\ ${ }^{2}$ GSI Helmholtzzentrum für Schwerionenforschung, 64291 Darmstadt, Germany \\ ${ }^{3}$ Helmholtz Institute Mainz, 55099 Mainz, Germany \\ ${ }^{4}$ Australian National University, Canberra, ACT 2601, Australia
}

\begin{abstract}
The new superheavy elements with $Z=113,115,117$, and 118 were recently accepted into the periodic table and have been named. Elements with $Z \geq 112$ are predominantly produced in ${ }^{48} \mathrm{Ca}$-induced fusion reactions on actinide targets. This pathway is exhausted at $Z=118$ due to the lack of target materials with sufficiently high proton number to reach elements with $\mathrm{Z} \geq 119$. Search experiments for yet heavier elements were performed at GSI Darmstadt and FLNR Dubna. The reactions ${ }^{50} \mathrm{Ti}+{ }^{249} \mathrm{Bk}$, which leads to $\mathrm{Z}=119$, as well as ${ }^{64} \mathrm{Ni}+{ }^{238} \mathrm{U},{ }^{58} \mathrm{Fe}+{ }^{244} \mathrm{Pu},{ }^{54} \mathrm{Cr}+{ }^{248} \mathrm{Cm}$, and ${ }^{50} \mathrm{Ti}+{ }^{249} \mathrm{Cf}$, leading to $\mathrm{Z}=120$, have been studied. Despite a total duration of these experiments of more than one year, neither succeeded in the identification of a new element. To obtain improved guidance for better-informed search experiments, nuclear reaction studies appear necessary and have recently started. Also technical advances will be an important pillar to this end. At GSI, work towards a new continuous-wave linear accelerator is ongoing and is briefly described.
\end{abstract}

\section{Introduction}

Recently, new elements with $\mathrm{Z}=113,115,117$, and 118 were officially accepted $[1,2]$ and were named in November 2016 [3]. With this, the periodic table of the elements (cf. Fig. 1) is complete to the end of the $7^{\text {th }}$ period. Fig. 2 shows the corresponding cut-out of the chart of nuclei (with decay properties updated according to [4]), where all known or claimed nuclei are indicated in colored boxes, overlaying the calculated shell correction energy landscape according to a macroscopic-microscopic model [5]. Also indicated (by white/black boxes for yet unknown isotopes) are the hypothetical decay chains originating from the $3 \mathrm{n}$ and $4 \mathrm{n}$ compound nucleus $(\mathrm{CN})$ evaporation channels following complete fusion of ${ }^{50} \mathrm{Ti}+{ }^{249} \mathrm{Bk}$ leading to ${ }^{299} 119^{*}$ (red bordered boxes), ${ }^{50} \mathrm{Ti}+{ }^{249} \mathrm{Cf}$ leading to ${ }^{299} 120^{*}$ (light blue bordered boxes), and ${ }^{54} \mathrm{Cr}+$ ${ }^{248} \mathrm{Cm},{ }^{58} \mathrm{Fe}+{ }^{244} \mathrm{Pu}$, and ${ }^{64} \mathrm{Ni}+{ }^{238} \mathrm{U}$, which all lead to ${ }^{302} 120^{*}$ (dark blue bordered boxes). Decay modes of unknown isotopes were assumed based on [6-8].

\section{The search for new elements beyond Og}

For the heaviest currently known elements with $\mathrm{Z}=112$ 118, employing fusion-evaporation reactions induced by doubly-magic neutron-rich ${ }^{48} \mathrm{Ca}$ on targets of ${ }_{92} \mathrm{U}-{ }_{98} \mathrm{Cf}$ [10] is well established, having been confirmed in multiple laboratories (e.g., [11-16]) and would, therefore, also appear to be a natural choice for attempts to synthesize yet heavier elements. Cross sections stay relatively constant

\footnotetext{
^e-mail: duellmann@uni-mainz.de
}

at a level of 0.5-10 pb over an extended region, suggesting that the accumulated data set provide a good basis for theoretical models to predict values for elements 119 and 120. The one caveat is that the corresponding target materials would have to be ${ }_{99} \mathrm{Es}$ and ${ }_{100} \mathrm{Fm}$, which are not available in quantities that suffice to make targets for such synthesis experiments. Current technology for the production of transuranium isotopes, e.g., at the ORNL's High Flux Isotope Reactor (HFIR) is capable of producing $\mu \mathrm{g}$ and pg amounts of long-lived ${ }_{99}$ Es and ${ }_{100} \mathrm{Fm}$ isotopes, respectively [17], which is orders of magnitude less than the experiments discovering and studying the heaviest known elements required. Still, fusion reactions with actinide targets are generally expected to provide the highest cross sections for the synthesis of new elements with $\mathrm{Z} \geq 119$. Thus, reactions induced by beams heavier than ${ }^{48} \mathrm{Ca}$ will be needed. Five of the most commonly suggested reactions giving access to $\mathrm{Z} \geq 119$ have been studied experimentally and are listed in Table 1 . None of these experiments have led to the conclusive discovery of a new element, though.

A total of more than one year of accelerator beamtime proved insufficient for the discovery of a new element, whereas any of the known elements would have been synthesized in significant amounts given the current sensitivity levels of such experiments. This suggests that either cross sections are significantly lower for all of the actinide-based reactions using beams heavier than ${ }^{48} \mathrm{Ca}$, or that some aspects relevant for performing a successful search experiment are not understood well enough. Likely, it is a combination of both. One such crucial aspect is 


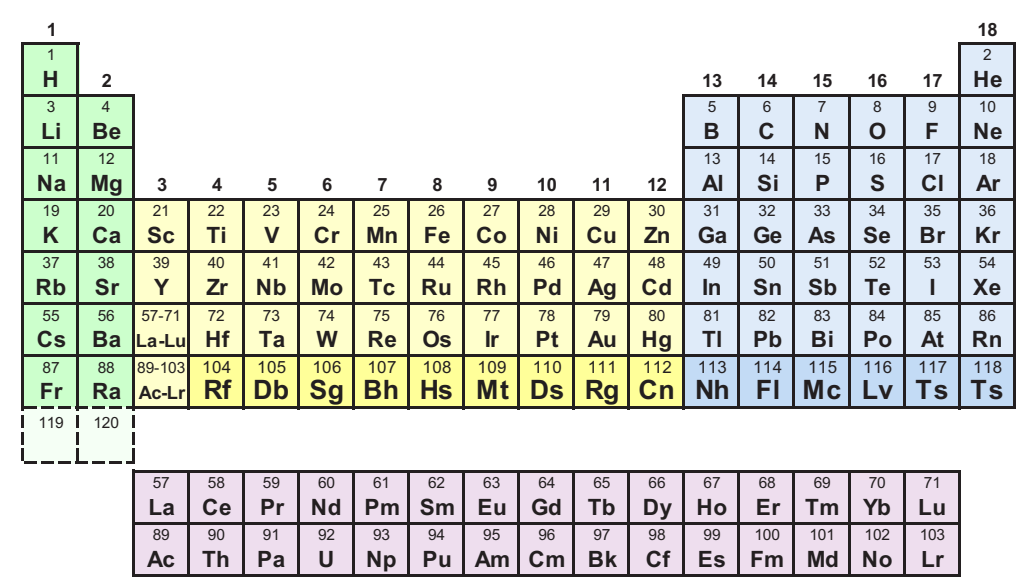

Figure 1. Periodic Table of the Elements. The known superheavy elements with $Z=104-118$ are highlighted. The positions of the elements with $\mathrm{Z}=119$ and 120 , which are yet unknown and would form the beginning of the $8^{\text {th }}$ period, are also indicated.

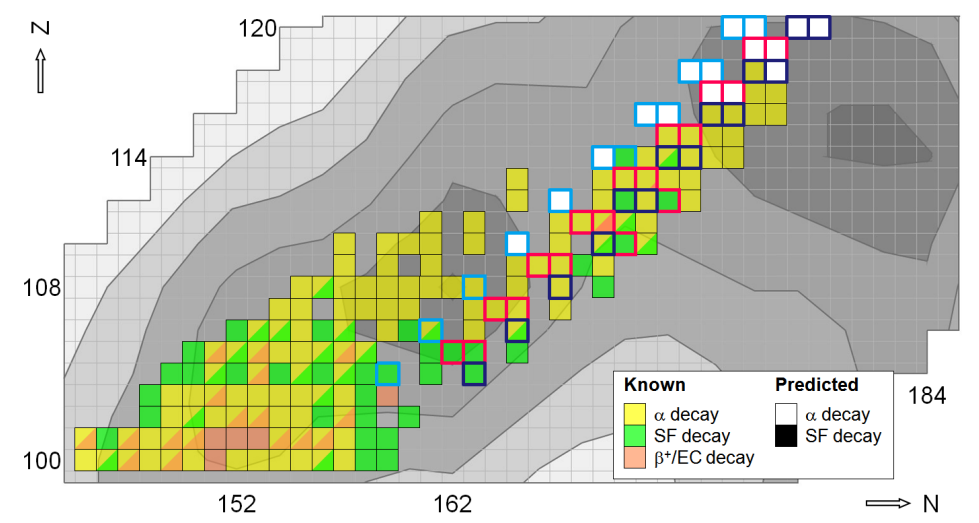

Figure 2. Chart of nuclei of the heaviest elements. The underlying contour plot shows calculated shell correction energies [5]. Known nuclei are indicated by colored boxes, new nuclei occurring in the hypothetical $3 \mathrm{n} / 4 \mathrm{n}$ decay chains produced in complete fusion of different target/projectile combinations leading to $\mathrm{Z}=119$ and $\mathrm{Z}=120$ are indicated by white and black boxes. See text for more details. Taken from [9].

Table 1. Fusion reactions employed to search for elements beyond $\mathrm{Z}=118$.

\begin{tabular}{ccccc}
\hline & $\begin{array}{c}\text { Compound } \\
\text { nucleus }\end{array}$ & $\begin{array}{c}\text { Beamtime } \\
\text { invested }\end{array}$ & $\begin{array}{c}\text { Cross section } \\
\text { limit }^{a}\end{array}$ & Ref. \\
\hline${ }^{50} \mathrm{Ti}+{ }^{249} \mathrm{Bk}$ & ${ }^{299} 119^{*}$ & 4.5 months & $b$ & {$[18]$} \\
${ }^{64} \mathrm{Ni}+{ }^{238} \mathrm{U}$ & ${ }^{302} 120^{*}$ & 4 months & $\geq 90 \mathrm{fb}$ & {$[19]$} \\
${ }^{58} \mathrm{Fe}+{ }^{244} \mathrm{Pu}$ & ${ }^{302} 120^{*}$ & 2 months & $\geq 400 \mathrm{fb}$ & {$[20]$} \\
${ }^{54} \mathrm{Cr}+{ }^{248} \mathrm{Cm}$ & ${ }^{302} 120^{*}$ & $>1$ month & $\geq 580 \mathrm{fb}$ & {$[21]$} \\
${ }^{50} \mathrm{Ti}+{ }^{249} \mathrm{Cf}$ & ${ }^{302} 120^{*}$ & $>1$ month & $b$ & {$[18]$} \\
\hline${ }^{a} 63.2 \%$ confidence level ("one-event limit") \\
$b$ \\
under final analysis
\end{tabular}

that of the optimum beam energy, which indeed seems to be understood insufficiently, as is detailed elsewhere [18]. One has to bear in mind that some of the predictions of the nuclear half-lives of the produced isotopes, especially in the case of $Z=120$, suggest these to be in the order of microseconds, i.e., comparable to the flight-time through the used separators. This may imply that losses due to decay-in-flight might have negatively affected ex- periments. Still, the predominant reason for the lack of finding any new element seems to be insufficient sensitivity. Preparations for improved experiments are ongoing at several laboratories. Thus, it appears appropriate to reflect on which aspects should get most attention to perform the best possible experiments, thus maximizing the likelihood of success. Obvious, purely technical aspects include higher primary beam intensities, targets that can withstand 
such intensities over extended periods [22, 23], more efficient separators, more sensitive detection systems with dead-time free data acquisition [24, 25] that allow safe identification of single decay chains from new elements, and longer irradiation times. As detailed in [18], where the system ${ }^{50} \mathrm{Ti}+{ }^{249} \mathrm{Cf}$ is employed to illustrate the current situation, optimum beam energies suggested by different theoretical frameworks differ by significantly larger amounts than can be covered in a single experiment. This highlights the need for a well-informed choice of the irradiation energy. Performing searches that are significantly more sensitive than those summarized in Tab 1 at multiple beam energies (which were all obtained at one single energy each) would consume years of beamtime.

In this situation, performing complementary experiments studying these fusion reactions, which yield information that guides future new element search efforts, appears well justified. For this, we recall that the formation of a superheavy nucleus is parametrized as the product of three individual steps, each characterized with its own probability: $\sigma_{E R}=\sigma_{c a p} \cdot \mathrm{P}_{C N} \cdot \mathrm{W}_{\text {sur }}$ with $\sigma_{E R}$ the evaporation residue (ER) cross section, $\sigma_{\text {cap }}$ the capture cross section, $\mathrm{P}_{C N}$ the compound nucleus formation probability, and $\mathrm{W}_{\text {sur }}$ the exit channel survival probability. The formation of superheavy elements is significantly suppressed by the quasifission $(\mathrm{QF})$ process, in which two colliding nuclei reseparate on a fast time-scale before coalescing into a compound nucleus, see [26], and Fig. 4 for a schematic of the relevant processes.

With QF being the complementary process to compound nucleus formation, $\mathrm{P}_{Q F}=1-\mathrm{P}_{C N}$, where $\mathrm{P}_{Q F}$ can reach large values. The $\mathrm{QF}$ process thus profits from high rates for systems where $\mathrm{P}_{C N}$ is small, e.g., SHE formation. Its study thus gives access to phenomena relevant for SHE formation, but in comparatively very short experiments. Observables include mass angle distributions [27] or mass energy distributions [28]. Combined with the corresponding experimental data, which can be recorded in short experiments, this will allow for the testing of different models, thus providing guidance concerning which ones deliver the most trustworthy predictions. Better informed selection of, e.g., the optimum irradiation energy will be invaluable. First steps in this direction have been made recently $[26,29]$. A combined effort encompassing theoretical as well as experimental work will provide an improved basis for the certainly coming future efforts to discover new elements with $\mathrm{Z}>118$ and to thus open a new row in the Periodic Table of the Elements.

\subsection{R\&D activities towards a new cw linac}

Superheavy element research is a pillar of the GSI research program for many decades and has led to high-visibility results. These include the discovery of six new elements [30] and chemical investigations for the first classification of new elements in the periodic table [31]. Technical advancements recently facilitated further milestone successes, including synthesis of elements up to Ts [12$14,32]$, extensive systematic nuclear structure studies of odd-mass even- $\mathrm{Z}$ isotones ( $\mathrm{N}$ constant) and odd-mass odd$\mathrm{Z}$ isotopes (constant $\mathrm{Z}$ ) in the "high-cross section" region $\mathrm{Z}=102-106$ around $\mathrm{N}=152$ (see [33] for a recent example) as well first such studies in Mc decay chains [13], highprecision mass measurements of single atoms of the heaviest elements [34, 35], laser spectroscopic studies of their electron shell [36], as well as advances in chemical studies up to the heaviest studied elements [37], resulting in a worldwide unique breadth and depth of the research program. Beams for all these studies are provided by the UNILAC linear accelerator, a pulsed machine that provides up to three types of projectiles from different ion-sources to several experiments in quasi-parallel operation. The maximum duty cycle is $25 \%$. This offers unique possibilities to significantly enhance beam availability by the installation of a continuous-wave (cw) heavy-ion linac for highly intense beams, as has been suggested, e.g., in [38]. With the foundation of the Helmholtz Institute in Mainz, Germany, the R\&D towards such a machine has started [39]. With its continuous beam, a cw machine would be ideal for a steady irradiation at significantly higher intensities than are presently available and will thus allow advancing the GSI programs in superheavy element research, materials research and biophysics. In the field of SHE, this would provide for experiments at higher sensitivity, as well as for in-depth studies of nuclei produced with higher cross sections thanks to higher production rates, Currently, a first "crossed H-bar" $(\mathrm{CH})$ type cavity has been built to demonstrate the performance of this novel accelerating structure and is in the commissioning phase. Fig. 5 shows a photograph of the cavity embedded by two sc solenoids mounted on a common support structure, which will be placed inside the cryostat.

Details on the cw linac demonstrator as well as a possible layout of the full machine are given in [40]. Next steps, which have started, include the installation of a string of cavities.

\section{References}

[1] P. J. Karol et al., Pure Appl. Chem. 88, 139 (2016).

[2] P. J. Karol et al., Pure Appl. Chem. 88, 155 (2016).

[3] L. Öhrström, and J. Reedijk, Pure Appl. Chem. 88, 1225 (2016).

[4] U. Forsberg et al., Nucl. Phys. A 953, 117 (2016).

[5] Z. Patyk, R. Smolanczuk, and A. Sobiczewski, Nucl. Phys. A 626, 337c (1997).

[6] I. Muntian, Z. Patyk, and A. Sobiczewski, Phys. At. Nucl. 66, 1015 (2003).

[7] I. Muntian et al., Acta Phys. Pol. B 34, 2073 (2003).

[8] R. Smolańczuk, J. Skalski, and A. Sobiczewski, Phys. Rev. C 52, 1871 (1995).

[9] Ch. E. Düllmann, EPJ Web Conf. 131, 08004 (2016).

[10] Y. T. Oganessian, and V. K. Utyonkov, Nucl. Phys. A 944, 62 (2015).

[11] S. Hofmann et al., Eur. Phys. J. A 32, 251 (2007).

[12] S. Hofmann et al., Eur. Phys. J. A 48, 62 (2012).

[13] D. Rudolph et al., Phys. Rev. Lett. 111, 112502 (2013). 


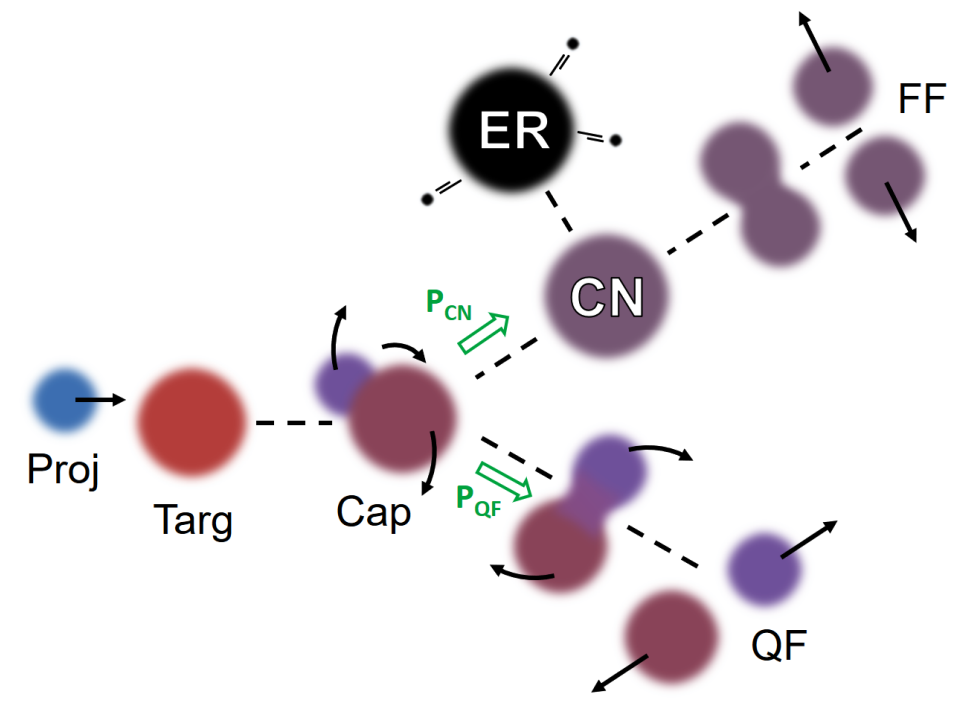

Figure 3. Schematic of the relevant processes in nucleus-nucleus collisions aimed at synthesizing the heaviest elements. Proj: projectile; Targ: target; Cap: captured system; CN: compound nucleus; ER: evaporation residue; FF: fusion-fission; QF: quasifission; $\mathrm{P}_{C N}$ : probability for the captured system to form a compound nucleus; $\mathrm{P}_{Q F}$ : probability for the captured system to fission. Obviously, $\mathrm{P}_{Q F}=1-\mathrm{P}_{C N}$.

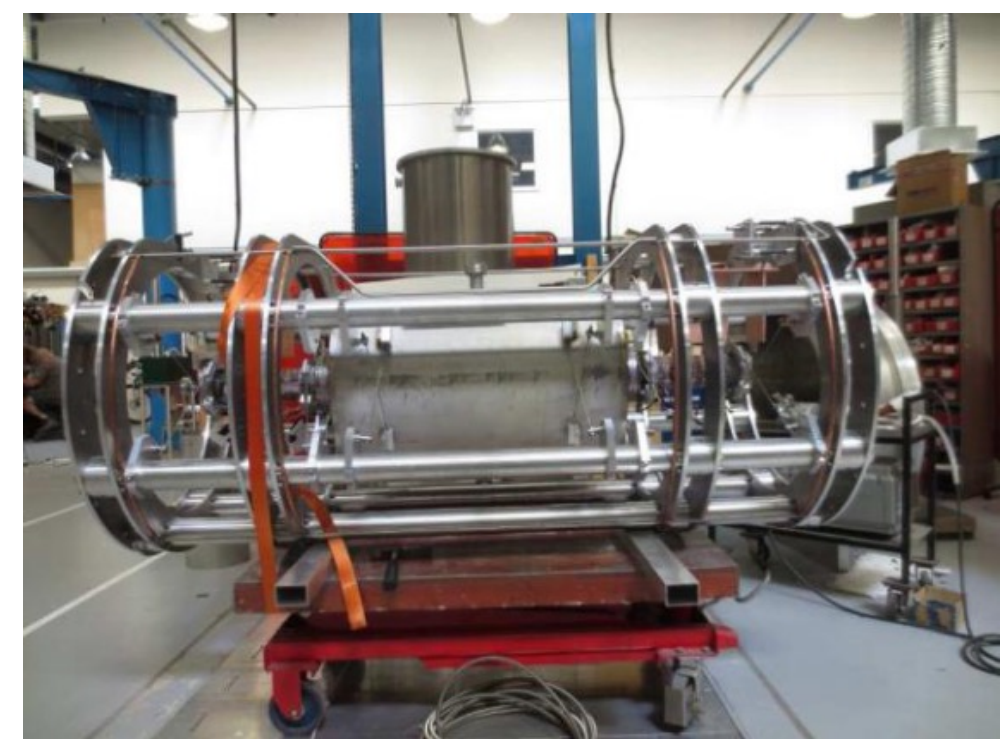

Figure 4. Superconducting $\mathrm{cw}$ linac demonstrator comprising a $\mathrm{CH}$ cavity embedded by two sc solenoids on a support frame (taken from [40]).

[14] J. Khuyagbaatar et al., Phys. Rev. Lett. 112, 172501 (2014).

[15] L. Stavsetra et al., Phys. Rev. Lett. 103, 132502 (2009).

[16] D. Kaji et al., J. Phys. Soc. Jpn. 86, 034201 (2017).

[17] J. B. Roberto et al., Nucl. Phys. A 944, 99 (2015).

[18] Ch. E. Düllmann et al., (in preparation) (2016).

[19] S. Hofmann, J. Phys. G 42, 114001 (2015).

[20] Y. T. Oganessian et al., Phys. Rev. C 79, 024603 (2009).

[21] S. Hofmann et al., Eur. Phys. J. A 52, 180 (2016).
[22] S. N. Dmitriev, and A. G. Popeko, J. Radioanal. Nucl. Chem. 305, 927 (2015).

[23] E. Jäger et al., J. Radioanal. Nucl. Chem. 299, 1073 (2014).

[24] I. H. Lazarus et al., IEEE Trans. Nucl. Sci. 48, 567 (2001).

[25] J. Khuyagbaatar et al., Phys. Rev. Lett. 115, 242502 (2015).

[26] D. J. Hinde et al., EPJ Web Conf. 131, 04004 (2016).

[27] R. du Rietz et al., Phys. Rev. C 88, 054618 (2013).

[28] M. G. Itkis et al., Nucl. Phys. A 944, 204 (2015).

[29] H. M. David et al., (in preparation) (2016). 
[30] G. Münzenberg, Nucl. Phys. A 944, 5 (2015).

[31] M. Schädel, Eur. Phys. J. D 45, 67 (2007).

[32] Ch. E. Düllmann et al., Phys. Rev. Lett. 104, 252701 (2010).

[33] S. Antalic et al., Eur. Phys. J. A 51, 41 (2015).

[34] M. Block et al., Nature 463, 785 (2010).

[35] E. Minaya Ramirez et al., Science 337, 1207 (2012).
[36] M. Laatiaoui et al., Nature 538, 495 (2016).

[37] A. Yakushev et al., Inorg. Chem. 53, 1624 (2014).

[38] S. Hofmann et al., in EXON 2004, edited by Y. E. Penionzhkevich, and E. A. Cherepanov (World Scientific, Singapore, Peterhof, Russia, 2004), pp. 157.

[39] S. Minaev et al., Phys. Rev. ST Accel. Beams 12, 120101 (2009).

[40] W. Barth et al., EPJ Web Conf. 138, 01026 (2017). 